\title{
Veracidad y Exactitud en "La Florida del Inca"
}

\author{
Por José Durand
}

\begin{abstract}
Fuentes e informantes. Cuando Garcilaso cuenta que recibió las relaciones de Carmona y Coles después de que "había acabado de escribir esta historia", habla bajo palabra de honor: ninguna prueba ofrece de ello y, por lo demás, tampoco tenía por qué ofrecerla, dentro de las costumbres de la época. Aquí los hechos parecen comprobar, una vez más, la veracidad de las afirmaciones de Garcilaso. Quienes, como antaño Manuel González de la Rosa, o más modernamente Roberto Levillier, tienen al Incal porl hombre poco fídedigno (1), de ningún modo querrían aceptar tales noticias Errada cautela. En general, sería fácil poner en duda la seriedad de las fuentes que tuvo Garcilaso para la Florida. Obra compuesta muchos años después de ocurridos los hechos que narra, la mayor parte del texto no se basa en testimonios hoy conocidos, sino en las informaciones verbales de un amigo del autor; luego, en dos crónicas hoy desaparecidas y también, indirectamente, en un tercer documento, que Garcilaso no conoció, pero que fué cotejado con parte de la Florida por un cronista amigo. Según indica el Proemio, la historia se funda y confirma en esos testimonios, de los cuales Garcilaso, muy extrañamente, da escasos datos: ni el nombre del principal informante, ni el del cronista que hizo el cotejo, y si bien da algunas noticias de las relaciones de Carmona y de Coles, hoy sólo las co-
\end{abstract}

(1) Los infortunados artículos de González de la Rosa aparecieron en la "Revista Histórica", Lima, 1907-1909, vols. ii - iv. Cf. también Roberto Levillier, Don Francisco de Toledo, supremo organizador del Perú, Buenos $\mathrm{Ai}$ res, 1942, vol. III, lib. I. 
nccemos a trcivés de la misma Florida. Todo, pues, lo sustenta el Inca con sólo empeñar su paiabra, y ni siquiera tiene a bien recordar el nombre del impresor cordobés en cuya casa se encontraba, roída de ratones, la crónica de Juan Coles. ¡Curiosa inclinación del Inca a la elusión y el silencio, muy propia ciertamente de su raza!

Sin embargo, no hay por qué desconfiar, pues la posteridad ha ratificado las afirmaciones de Garcilaso. Hoy se sabe que el informante principal se llamaba Gonzalo Silvestre, intimo amigo del Inca y miembro de la expedición de Soto (2). Se sabe también que Carmona vivió en Priego, pueblo cercano a los de Garcilaso y Silvestre (3), y que murió en 1591. Pocas noticias existen del soldado Coles, pero en cambio parece que Garcilaso tenía amistad con varios impresores cordobeses, alguno de los cuales pudo ser el que le proporcionó el manuscrito: uno, Francisco de Cea, a quien por cierto hizo un pequeño legado en su testamento (4); otro, la viuda de Andrés de la Barrera, la cual editó la segunda parte de los Comentarios. De otro lado vemos que el cronista que hizo el cotejo de la Florida con ciertos documentos que poseía ha resultado ser el doctor Ambrosio de Morales quien, como cronista regio, disponía de grandes fondos documentales (5). Todo, pues, parece confirmar la honradez y la exactitud de las noticias que da el autor sobre las principales fuentes de su obra.

No hay por qué dudar de la veracidad de Garcilaso, hombre que estimaba en mucho la honra (6) C $y$ que en Gonzalo Silvestre, típico hidalgo español, pondẹraba eluquẻnsel lipreciaserde "decir verdad en

(2) Cf. José de la Riva-Agüero, La historia en el Perú, Lima, 1910, pp. 43-44. Cf. también el proemio de la Florida; Rubén Vargas Ugarte, S. J., Nota sobre Garcilaso, en "Mercurio Peruano, 1930, núm. 137-138, pp. 106107; y Raúl Porras Barrenechea, Una joya bibliográfica peruana, en "El Comercio", Lima, 15-17 de septiembre de 1948.

(3) Montilla, en donde residía el Inca, está a mitad de camino entre Priego y Córdoba.

(4) Cf. J. D. Un sermón editado por el Inca Garcilaso, en Homenaje a Amado Alonso, "Nueva Revista de Filología Hispánica", México, 1953, p. 594 , t. y n. 3.

(5) Cf. Asensio, loc. cit.; el documento que poseía Ambrosio de Morales reunía las declaraciones hechas en México, acatando órdenes del virrey Antonio de Mendoza, por los sobrevivientes de la hueste de Soto.

(6) Cf. J. D. La idea de la honra en el Inca Garcilaso, en "Cuadernos Americanos", México, noviembre-diciembre de 1951, pp. 194-213; reproducido en "Panorama", Washington", núm. 1, 1953, pp. 67-83. 
toda cosa" (7). A la luz de repetidas comprobaciones de detalle, hoy es preciso aceptar la hidalga honradez de las afirmaciones de Garcilaso. Gran parte de las tachas de falsedad, especialmente dentro de las lanzadas por Roberto Levillier, se basan en la tesis que sostiene Garcilaso de que los incas no practicaban ritos de sacrificios humanos; sin embargo, eso lo creía de buena fe, y en anotaciones marginales al ejemplar de la Historia de Gómara que poseyó, escritas con la espontaneidad indudable de quien hace apuntes para sí, sostiene rotundamente la misma afirmación que luego sostendrá en los Comentarios reales. Nueva prueba, entre otras muchas que podrían darse, de que la reputación de Garcilaso como autor veraz debe quedar fuera de duda.

Si el Inca fué hombre serio y fidedigno, merecedor del respeto de quienes lo trataron, y si, como se ha visto, no mintió al subrayar el valor de las fuentes en que se basa su historia de la Florida, tampoco es de suponer que mintiese al apuntar que, cuando recibió las relaciones de Carmona y Coles, ya estába escrita su obra. Además, esta afirmación, hecha "bajo palabra" como las otras, se confirma en la noticia que el mismo Garcilaso había dado en 1589 a Felipe II, de que por entonces estaba sacando "en limpio" la historia. Y cuando, años más tarde, Garcilaso la da otra vez por terminada, lo hace, sin duda alguna, porque la ha vuelto a "escribir de nuevo" (segunda redacción), después de recibir esás relaciones. Yeademás, no olvidemos que abundan testimonios de gue hacia 1602 , corrigió mucho la obra. Como se ve, siempre había hablado con entera honradez.

Protestas de veracidad. Casi siempre que un pasaje resulta extraño al lector, el Inca sale al encuentro de la duda. Así por ejemplo, en el libro IV, cap. XIV, Garcilaso narra una hazaña de Gonzalo Silvestre, quien de un tajo partió en dos por la cintura a un indio de la provincia llamada Tula. Más adelante, en el libro VI, cap. XIX, vuelve a ocuparse del asunto, confirmándolo, y refiere que, ya vueltos los expedicionarios a México, el factor Gonzalo de Salazar supo allí por otros soldados, la hazaña de Silvestre, "y viendo la espada, que era antigua, de las que ahora llaman viejas, se la pidió para ponerla en su recámara, por joya de mucha estima". En muchos casos, las

(7) Cf. Porras Barrenechea, Una joya ..., loc. cit.; de un examen de las apostillas manuscritas se deduce, bien a las claras, que la creencia de Garcilaso era absolutamente sincera. ¡Qué lejos estamos de las indignadas palabras que contra el Inca lanzaba González de la Rosa o Levillier! 
noticias sorprendentes que refiere encuentran confirmación en otras crónicas, las cuales el Inca no conoció. Cuando encarece el valor de los indios, o bien la extremada cortesía de algunos caciques, se siente obligado a insistir en su veracidad, temiendo el recelo del lector; sin embargo, las crónicas del Hidalgo de Elvas, Rodrigo de Rangel y el factor Luis Hernández de Viedma coinciden largamente con Garcilaso en ambos puntos; Rangel, por ejemplo, escribe que en la región de Apalache "acaecieron muchas cosas notables con los indios, los cuales son valentísimos hombres" (8), y el Hidalgo de Elvas refiere, coincidiendo con Garcilaso, cómo dos caciques riñeron al sentarse a la mesa con Soto "sobre debía sentarse a la mano derecha" (9).

En más de una ocasión, Garcilaso llega a defender su historia con solemnes juramentos. Al elogiar las virtudes ciudadanas de los indios floridos, confiesa "que muchas veces me pesó hallarlas en el discurso de la historia tan políticas, tan magníficas y excelentes, por que no se sospechase que eran ficciones mías y no cosecha de la tierra. De lo cual me es testigo Dios Nuestro Señor que no solamente no he añadido cosa alguna a la relación que se me dió, antes confieso, con vergüenza y confusión mía, no haber llegado a magnificar las hazañas como me las recitaron que pasaron en efecto, de que pido perdón a todo aquel reino y a los que leyeren este libro. $Y$ esto baste -añade- para qüel seo de el crêditō que se debe a quien, sin pretensión de interés. . tomó el trabajo de escribir esta historia" (10). El pasaje, sin duda iardios (11), nos presenta ap Ghca en el momento en que se empeña en disipar dudas, movido probablemente por las que algunos contemporáneos debieron formularle: quizá los mismos censores. El temor de ser tachado de historiador parcial a causa de su condición de indio, lo tuvo, como se ve, desde que empezaba a componer su obra; pero ese temor debió adquirir fundamento cuando años después, al corregir la historia, creía necesario añadir pasajes aclaratorios. En otra ocasión, al relatar la hazaña de los treinta caballeros con quienes Soto envió un mensaje a Pedro Calderón, escribe: "Pues en ley de hijodalgo afirmamos con verdad que en siete días anduvie-

(8) Apud Gonzalo Fernández de Oviedo, General y natural historia de las Indias, lib. XVII, cap. xxiv.

(9) Hidalgo de Elvas, Relacam Verdadeira... Evora, 1557; reimpresa en Lisboa, 1844; cf. el cap. xxiv.

(10) Cf. Florida, lib. VI, cap. xxi.

(11) Cf. supra, parag. 2, La redaeción: cronología. 
ron estos caballeros ciento y siete leguas, una más o menos" (12). Y cuando se dispone a referir las curiosas anécdotas del soldado Sanjurgem advierte: "Por haber sido hombre notable, será razón digamos algunas cosas suyas en particular, pues todas son de nuestra historia; y porque son extraordinarias, remito lo que sobre ellas y sobre cualquiera otra cosa que aquí 0 en otra parte dijere, a la corrección y obediencia de la Santa Madre Iglesia Romana, cuyo catolicísimo hijo soy por la misericordia de Dios, aunque indigno de tal madre" (13). Juramentos como estos, hechos en nombre de Dios una vez, otra en ley de hijodalgo y por último empeñando su palabra de cristiano calólico, no parecen propios de un historiador mentiroso ni inseguro de la verdad de sus noticias. Sin embargo, a la vista de ello y sin advertir la conformidad general del Inca con otros cronistas, William Bancroft y Marcelino Menéndez Pelayo afirmaron que la Florida es ante todo una obra literaria (14), sumamente crédula y alejada de la realidad. En previsión de tales dudas, Garcilaso había certificado ya, siglos atrás su cuidado de ofrecer datos puntualmente ratificados por su informante y co-autor; sin alguna vez errase o no, es cosa que puede discutirse, pero siempre deberá reconocerse que la actividad de Garcilaso como historiador, lejos de ingenua y crédula, resulta ser escrupulosamente crítica.

La base histórica de la Florida, que tanto preocupó al Inca, descansa en la autoridad de su amigo Gonzalo-Silvestre. Aparte de la fe que tenía en ella,oGarcilasociaeconfirmó directamente, no sólo en el trato diario que mantuvieron al componer juntos la historia, sino también mediante la corroboración obtenida en otras fuentes, como las relaciones que poseín el doctor Morales, la de Alonso de Carmona y la de Juan Coles. Con todo, lo principal era subrayar enfáticamente la autenticidad de los datos de Silvestre. Cuando Garcilaso narra el heroísmo de un grupo de indios que permanecieron más de un día dentro de una laguna, por no caer en manos españolas, comenta: "Hazaña por cierto increíble y que yo no osara escribirla, si la autoridad de tantos caballeros no me la certificara, sin la autoridad y verdad

(12) Cf. Florida, lib. II, p. II, cap. xiii.

(13) Ibid., lib. V, p. II, cap. v.

(14) Cf. William Bancroft, History of the United States, vol. I, New York, 1834; Marcelino Menéndez Pelayo, ristoria de la poesia hispanoamericana, Madrid, vol. II, 1913, pp. 145 y ss. 
del que me dió la relación de esta historia", o sea Silvestre (15). Por lo demás, Rangel y el Hidalgo de Elvas coinciden en referir la hazaña de la laguna aun cuando discrepan sobre el lugar en que ella ocurrió (16). Al poner Garcilaso en boca de un indio un largo parlamento, lleno de corteses requerimientos, puntualiza: "Todas fueron palabras del indio, que no le añadimos alguna, más de pasarlas de su lengua a la española o castellana" (17). Significativamente, el Hidalgo de Elvas también reproduce o reconstruye parlamentos semejantes Y lo mismo hacen otros cronistas; pero, muy significativamente también, ninguno se sientê en la obligación de insistir como el Inca en la bondad de sus noticias: sabían la pura verdad y como tal la contaban ingenuamente, sin pensar sn la acogida que merecerían de un lector avisado. Garcilaso en cambio, consciente de su condición de indio y de primer historiador americano, sale al paso de cualquier duda con protestas de honradez.

Garcilaso comprendía muy bien que en Silvestre reposaba su propia autoridas y aun su reputación. Volviendo a nuestro primer propósito -escribe Garcilaso-- que es de certificar en ley de cristiano que escribimos verdad en lo pasado y, con el favor de la Suma Verdad, lo escribiremos en lo porvenir, diré lo que en este paso me pasó con el que me daba la relación. Al cual, si no lo tuviera por tan hijodalgo y fidedigno como lo es, como adelante on otros pasos diremos de su reputación, no presumiera yo que escribiera tanta verdad como la presumo y certifico pos ed 't cricuerita cauélalocomponer juntos la historia, Silvestre le ordenó: "Escribid sin escrúpulo alguno lo que os digo, créanlo o no lo crean, que con haber dicho verdad de lo que sucedió, cumplimos con nuestra obligación, y hacer otra cosa seríc hace agravio a las partes. Todo esto, ccmo lo he dicho, me pasó con mi autor, y yo lo pongo para que se entienda y crea cue presumimos escribir verdad, antes con falta de elegancia y relórica necesaria para poner las hazañas en su punto, que con sobra de encarecimiento, porque no lo alcanzó y porque adelante, en otras cosas tan grandes y mayores que veremos, será necesario reforzar la reputación de nues-

(15) Florida, lib. II, p. I, cap. xxv. Por este pasaje del Inca se sabe que interrogó a cuantas gentes conoció que hubieran estado en la jornada o que supiesen de ella.

(16) Según el Fidalgo de Elvas, la acción ocurrió en Napetaca, el 15 de septiembre de 1539 (cap. xi); la versión de éste se asemeja más a la de Garcilaso que la de Rangel.

(17) Cf. Florida, lib. II, p. I, cap. xxv; Hidalgo de Elvas caps. xvi, xvii y passim. 
tro crédito, no diré ahora más" (18). Importantísimo pasaje, que nos presenta en vivo todo el proceso crítico de discriminación de datos y elaboración de la obra, unidos el informante-cronista Gonzalo Silvestre y el cronista-historiador. Aquí, derechamente, responde Garcilaso a Menéndez Pelayo y Bancroft, para quienes la Florida es un continuo abandonarse a las bellezas literarias. Según esa clásica concepción que tenía Garcilaso de "poner las hazañas en su punto", la historia debía honrar y "magnificar" las grandezas del pasado, mediante los recursos de la retórica, siempre puestos al servicio de la verdad. Y en fin, vemos a Garcilaso actuar en dos etapas muy distintas de la composición de la Florida: una, cuando en unión de Silvestre escribe la primera redacción, y auque prevé dudas en el lector, se limita a establecer los hechos y a consignarlos; otra, cuando, al corregir la obra, después de los tropiezos habidos en la edición de ella, se afana por ganar crédito y para ello pondera repetidas veces las excelentes prendas y la autoridad de testigo que tenía su oculto informante, que hoy sabemos que es Gonzalo Silvestre.

Afán de exactitud. En repetidos pasajes de la Florida se aprecia la grave preocupación que tenía Garcilaso, como historiador severo que era, de ofrecer datos cabales, al menos hasta donde fuese posible. Esa actitud lo mueve a excusarse siempre que no consigue ser del todo preciso, aun cuando ello resultaba muy natural Su informante Gonzalo Silvestre tenía que operar con recuerdos de hechos muy viejos y así, cuando tenía que ofrecer referendias geográficas por ejemplo, sólo alcanzaba a hablar aproximadamente. Debe tenerse presente que cuando los restos de la hueste de Soto, a órdenes de Luis de Moscoso de Alvarado, salieron de la tierra, no llevaban ya "carta de marear, ni aguja, ni astrolabio para tomar el altura del sol, ni ballestilla para la del norte" (19); ni Silvestre, pues, ni ninguno de los otros cronistas de esa jornada, podía así ofrecer dalos exactos de rumbo y posición. Pero el Inca, movido de un afán científco no sólo de veracidad, sino de excctitud, lleva su honradez al máximo y advierte, por ejemplo, que "este rumbo y todos los demás que en esta historia se dijeren, es de advertir que no se tomen precisamente, para culparme si otra cosa pareciere después, cuando aquella tiera se ganare, siendo Dios Servido". No se olvide que, entre los fines de la Florida, se contaba el de incitar a los españoles a la conquista de la tierra, por lo cual Garcilaso deseaba ser de provecho en sus referencias. En otra ocasión insiste y

(18) Cf. Florida, lib. II, p. I, cap. xxvii.

(19) Ibid, lib. VI, cap. xi. 
dice que la empresa de esa conquista "se debe guardar para algún bien afortunado, que ial será el que hiciere, y entonces se verificarán las faltas de mi historia, de que he pedido perdón muchas veces" (20). Siempre puntual, apunta que "navegaron esios españoles muchas leguas mas no podemos decir cuántas, con grandísimo contento de tomar el río de Palmas", cosa que "certificaban los que se jactaban de cosmógrafos y grandes marineros; más en hecho de verdad, el que dia ellos más sabía no sabía en qué mar ni por cuál región navegaban" (21).

En su deseo de ser preciso, busca una justificación de no poder serlo y la ofrece con su habitual claridad de ideas: "Por ahora - -ss. cribe en un pasaje tardío, como suelen serlo éstos- yo no puedo verificar más esta relación de como la escribo, y aun ha sido mucho haber sacado en limpio esto poco al cabo de tantos años que ha que pasó, y por gente que su fin no era andar demarcando la tierra, aunque la andaban descubriendo, sino buscar oro y plata; por lo cual se podrá admitir en este lugar el descargo que en otros he dado de las faltas que esta historia lleva, en lo que toca a la cosmografía, que yo quisiera haberla escrito muy cumplidamente, para dar mayor y mejor noticia de aquella tierra" (22). ¡Qué alejadas están estas palabras de las que escribiría un simple literario, o un historiador ingenuo, como pensaba el viejo Bancroft! Por el contrario, la formación humanísticaldellinca, Cespaciosamentel Gdquirida y admirablemente lograda, apōrece aquuíc en continnoo ejercicio, aplicándose tanto en los trabajos preparatorios y en la primera redacción como en el momento de la autocrítica, cuando hace las correcciones finales. Y en fin, esa misma actitud de historiador escrupuloso aparece también en el cuidado con que Garcilaso coteja las noticias de Silvestre sobre las tierras de Apalache, con las que de ellas había dado, tiempo atrás, Alvar Núñez Cabeza de Vaca; para Silvestre, eran campos feraces y según los Naufragios, paupérimos. Y el Inca, deseoso siempre de armonizar, evitando la tacha de falso para Alvar Núñez, piensa que éste debió haber pasado por otro lugar de la misma provincia (23). Todo el espíritu del Inca está aquí, tanto en el cuidado de no dañar la honra ajena como en la firme voluntad de establecer los hechos con perfecta claridad.

(20) Ibid., lib. II, p. I, cap. xii.

(21) Ibid., lib. VI, cap. ix.

(22) Ibid., cap. xii.

(23) Ibid., cap. viii. 